\title{
O Princípio de Saint-Venant em Elasticidade Não Linear
}

J.D. da SILVA, J.A. FERREIRA, Departamento de Matemática, UFES, 29060-900 Vitória, ES, Brasil.

I.-S. LIU, Instituto de Matemática, UFRJ, 21945-970 Rio de Janeiro, RJ, Brasil.

Resumo. Neste trabalho, estendemos os resultados obtidos por Horgan e Payne [6] em 1992, e demonstramos que o Princípio de Saint-Venant é verdadeiro para um corpo ocupando a região retangular $\Omega=\left\{\left(x_{1}, x_{2}\right) \mid 0<x_{1}<l, \quad-h / 2<x_{2}<h / 2\right\}$ onde $l \gg h$, do plano Cartesiano, em material elástico, cuja equação constitutiva é uma generalização da Lei de Hooke com termos não lineares em pequenas deformações. Os termos não lineares considerados pelos citados autores são pelo menos de terceira ordem. A inclusão dos termos de segunda ordem neste trabalho representa um modelo mais realístico. O resultado principal é estabelecido usandose técnicas de desigualdades diferenciais para funcionais quadráticos.

\section{Introdução}

Boussinesq [1], em 1885, foi o primeiro a tentar uma formulação e uma prova rigorosa para a conjectura elaborada por Sain-Venant [10] publicada em 1853, elevando a conjectura original ao posto de "Princípio", e muitos textos até hoje, dão Boussinesq como referência para a demonstração do Princípio de Saint-Venant. Entretanto, uma prova de que o resultado vale dentro da teoria linear só foi conseguida por Toupin [11] em 1965.

Resultados semelhantes ao Princípio de Saint-Venant, dentro de uma teoria restrita da elasticidade não linear, foram obtidos por Roseman [9], Roseman e Breuer [2], Horgan e Knowles [3, 4], Horgan e Payne [5, 6], todos sob a hipótese de pequenas deformações.

O objetivo principal deste trabalho é fazer uma extensão dos resultados obtidos por Horgan e Payne [6], e demonstrar que o Princípio de Saint-Venant é verdadeiro para um material elástico ocupando uma região retangular, utilizando para isso uma equação constitutiva não linear considerando pequenas deformações como uma generalização natural da Lei de Hooke. Esta equação contém termos não lineares de segunda ordem; termos estes importantes e não considerados em [6].

Usaremos a convenção de somatório para simplificar expressões, ou seja, quando um par de índices aparece numa expressão significa um somatório sobre o índice de 1 a 2 , no caso bidimensional. Com esta convenção, por exemplo, $\left[u,{ }_{i j}\right],{ }_{i j}=0$, onde a vírgula representa a derivada parcial, é a clássica equação biharmônica. 


\section{Formulação do Problema}

De acordo com a teoria da elasticidade linear, o estudo das deformações planas de uma região $\Omega$, quando não existem forças de volume atuantes, se reduz a encontrar uma solução $\phi$ da equação biharmônica, que satisfaça às condições de contorno do problema, onde $\phi$ é a função de tensão de Airy, em termos da qual as tensões $\tau_{i j}$ são dadas por $\tau_{11}=\phi, 22, \quad \tau_{22}=\phi,{ }_{11}, \quad \tau_{12}=-\phi,{ }_{12}$. A existência de uma função de Airy implica que a equação de equilíbrio, isto é, $\tau_{i j}{ }_{j}=0$, seja satisfeita identicamente.

Estamos interessados nas deformações planas na teoria de elasticidade não linear de um corpo elástico, ocupando a região retangular $\Omega$ do plano Cartesiano definida por $\Omega=\left\{\left(x_{1}, x_{2}\right) \mid 0<x_{1}<l,-h / 2<x_{2}<h / 2\right\}$, onde $l \gg h$.

Consideraremos um modelo constitutivo não linear da seguinte forma:

$$
e_{i j}=\frac{1+\nu}{\mathrm{E}} \tau_{i j}-\frac{\nu}{\mathrm{E}} \tau_{k k} \delta_{i j}+\frac{1}{\mathrm{E}} \mathcal{H}\left(\tau_{k l}\right) \tau_{i j},
$$

onde $\delta_{i j}$ é a função 1 e 0 , E é o módulo de Yong, $\nu$ módulo de Poisson e $\mathcal{H}$ é uma função de tensão $\tau_{k l}$, representando formalmente uma generalização da Lei de Hooke com termos não lineares ver [6] e [7].

Usando a função $\rho(\phi)$ definida por $\rho(\phi)=1+\mathcal{H}\left(\tau_{k l}\right)$, a equação de compatibilidade do tensor de deformação, isto é, $e_{11,22}+e_{22,11}-2 e_{12,12}=0$, se reduz numa equação diferencial parcial de quarta ordem para a função de Airy $\phi$,

$$
\left[\rho(\phi) \phi,{ }_{i j}\right],{ }_{i j}=0 \quad \text { em } \quad \Omega .
$$

Neste trabalho, consideremos a não-linearidade representada pela função $\mathcal{H}\left(\tau_{k l}\right)$ da seguinte maneira:

$$
\mathcal{H}\left(\tau_{k l}\right)=\lambda_{1} \tau_{k k}+\lambda_{2} \tau_{k k} \tau_{l l}+\lambda_{3} \tau_{k l} \tau_{k l}
$$

onde $\lambda_{1}, \lambda_{2}$ e $\lambda_{3}$ são parâmetros materiais. Assim estamos considerando a relação constitutiva da seguinte forma:

$$
e_{i j}=\frac{1+\nu}{\mathrm{E}} \tau_{i j}-\frac{\nu}{\mathrm{E}} \tau_{k k} \delta_{i j}+\frac{1}{\mathrm{E}}\left(\lambda_{1} \tau_{k k}+\lambda_{2} \tau_{k k} \tau_{l l}+\lambda_{3} \tau_{k l} \tau_{k l}\right) \tau_{i j} .
$$

Observe que a relação constitutiva (2.4) que será utilizada em nossas demonstrações é uma extensão da Lei de Hooke contendo termos não lineares de $2^{a}$ e $3^{a}$ ordem, enquanto no trabalho de Horgan e Payne [6] a relação constitutiva utilizada é

$$
e_{i j}=\frac{1+\nu}{\mathrm{E}} \tau_{i j}-\frac{\nu}{\mathrm{E}} \tau_{k k} \delta_{i j}+H(q),
$$

onde $q=\tau_{k l} \tau_{k l}$ e $H(q) \geq 0$. Apesar da sua generalidade, $H=\mathcal{H}\left(\tau_{k l}\right)$ é uma função somente de $q$, portanto, os termos não lineares são pelo menos de $3^{a}$ ordem. Assim, a relação constitutiva (2.4) é mais realista.

Assumiremos que uma solução $\phi$ da equação $(2.2)$ satisfaz $\phi \in \mathcal{C}^{4}(\Omega) \cap \mathcal{C}^{2}(\bar{\Omega})$, e usaremos a notação $\|\phi\|=\sum_{|\gamma| \leq 2} \sup \left\{\left|\partial^{\gamma} \phi(x)\right|: x \in \Omega\right\}$, onde $\partial^{\gamma}$ são as derivadas de ordem $\gamma$.

O objetivo deste trabalho é demonstrar que o Princípio de Saint-Venant é verdadeiro para corpos ocupando regiões retangulares e satisfazendo à hipótese constitutiva dada por (2.4) com $\lambda_{1}, \lambda_{2}$ e $\lambda_{3}$ suficientemente pequenos. 


\section{Condições na Fronteira}

Suponhamos que um corpo elástico ocupe a região $\Omega$ em equilíbrio, sob a ação das forças sobre a sua fronteira.

- O lado longo em $x_{2}=-h / 2$ está sujeito a tensões normal e cisalhante determinadas respectivamente por funções $q_{1}$ e $v_{1}$ contínuas em $(0, l)$, e o lado longo em $x_{2}=h / 2$ por funções $q_{2}$ e $v_{2}$ também contínuas em $(0, l)$.

- A extremidade em $x_{1}=0$ está sujeita a tensão normal e cisalhante determinadas respectivamente por funções $f_{1}$ e $g_{1}$ e a extremidade $x_{1}=l$ por funções $f_{2}$ e $g_{2}$. As funções $f_{1}, g_{1}, f_{2}$ e $g_{2}$ são contínuas em $[-h / 2, h / 2]$.

Temos, portanto, as seguintes condições na fronteira:

$$
\begin{array}{lll}
\tau_{12}\left(x_{1},-h / 2\right)=v_{1}\left(x_{1}\right), & \tau_{22}\left(x_{1},-h / 2\right)=q_{1}\left(x_{1}\right), & 0<x_{1}<l, \\
\tau_{12}\left(x_{1}, h / 2\right)=v_{2}\left(x_{1}\right), & \tau_{22}\left(x_{1}, h / 2\right)=q_{2}\left(x_{1}\right), & 0<x_{1}<l, \\
\tau_{11}\left(l, x_{2}\right)=f_{2}\left(x_{2}\right), & \tau_{12}\left(l, x_{2}\right)=g_{2}\left(x_{2}\right), & -h / 2 \leq x_{2} \leq h / 2, \\
\tau_{11}\left(0, x_{2}\right)=f_{1}\left(x_{2}\right), & \tau_{12}\left(0, x_{2}\right)=g_{1}\left(x_{2}\right), & -h / 2 \leq x_{2} \leq h / 2 .
\end{array}
$$

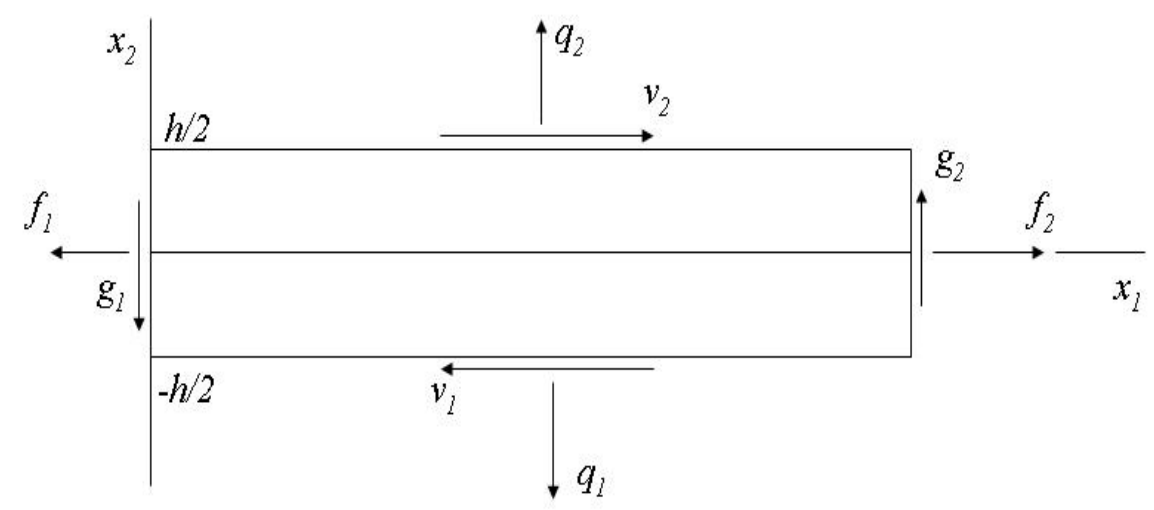

Para garantir a continuidade das tensões na fronteira, exigiremos que

$$
\begin{array}{ll}
\lim _{x_{1} \rightarrow 0} v_{2}\left(x_{1}\right)=g_{1}(h / 2), & \lim _{x_{1} \rightarrow l} v_{2}\left(x_{1}\right)=g_{2}(h / 2), \\
\lim _{x_{1} \rightarrow 0} v_{1}\left(x_{1}\right)=g_{1}(-h / 2), & \lim _{x_{1} \rightarrow 0} v_{1}\left(x_{1}\right)=g_{2}(-h / 2) .
\end{array}
$$

As condições de fronteira (2.6)-(2.9) em termos da função de Airy são dadas por

$$
\begin{array}{lll}
\phi,{ }_{11}\left(x_{1},-h / 2\right)=q_{1}\left(x_{1}\right), & \phi,{ }_{12}\left(x_{1},-h / 2\right)=-v_{1}\left(x_{1}\right), & 0<x_{1}<l, \\
\phi,{ }_{11}\left(x_{1}, h / 2\right)=q_{2}\left(x_{1}\right), & \phi,,_{12}\left(x_{1}, h / 2\right)=-v_{2}\left(x_{1}\right), & 0<x_{1}<l, \\
\phi,{ }_{22}=f_{2}\left(x_{2}\right), & \phi,,_{12}=-g_{2}\left(x_{2}\right), & -h / 2 \leq x_{2} \leq h / 2, \\
\phi,{ }_{22}=f_{1}\left(x_{2}\right), & \phi,,_{12}=-g_{1}\left(x_{2}\right), & -h / 2 \leq x_{2} \leq h / 2
\end{array}
$$


Observamos que não serão necessárias as formas explícitas das forças $q_{1}, q_{2}, v_{1}$, $v_{2}, f_{1}, f_{2}, g_{1}$ e $g_{2}$ para obter os resultados neste trabalho. Porém, as forças aplicadas sobre a fronteira têm que manter o corpo em equilíbrio.

\section{Problema de Valores na Fronteira}

Supondo a continuidade de $\phi, \partial \phi / \partial x_{i}$ e $\partial^{2} \phi / \partial x_{i}^{2}$ até à fronteira, concluimos que a distribuição de tensões na região $\Omega$, com as condições apresentadas, é dada pela solução do problema a seguir:

$$
\begin{aligned}
& {\left[\rho(\phi) \phi,{ }_{i j}\right],{ }_{i j}=0 \quad \text { em } \Omega,} \\
& \phi\left(x_{1},-h / 2\right)=F_{1}\left(x_{1}\right), \quad \phi,{ }_{22}\left(x_{1},-h / 2\right)=G_{1}\left(x_{1}\right), \quad 0<x_{1}<l, \\
& \phi\left(x_{1}, h / 2\right)=F_{2}\left(x_{1}\right), \quad \phi,{ }_{22}\left(x_{1}, h / 2\right)=G_{2}\left(x_{1}\right), \quad 0<x_{1}<l, \\
& \phi\left(l, x_{2}\right)=F_{3}\left(x_{1}\right), \quad \phi,{ }_{1}\left(l, x_{2}\right)=G_{3}\left(x_{2}\right), \quad-h / 2 \leq x_{2} \leq h / 2, \\
& \phi\left(0, x_{2}\right)=F_{4}\left(x_{2}\right), \quad \phi,{ }_{1}\left(0, x_{2}\right)=G_{4}\left(x_{2}\right), \quad-h / 2 \leq x_{2} \leq h / 2,
\end{aligned}
$$

onde as funções de $F_{1}$ a $G_{4}$ são obtidas de (2.12)-(2.15) por integração.

\section{O Princípio de Saint-Venant}

\section{Problema Referencial}

Daqui em diante denominaremos $u\left(x_{1}, x_{2}\right)$ uma solução conhecida do problema de valores na fronteira (2.16), (2.17)-(2.20), que pasaremos a denominar Problema referencial.

A forma explicita da solução $u\left(x_{1}, x_{2}\right)$ não será exigida para obter os resultados principais neste trabalho. Porém, vamos supor que a solução seja limitada por uma constante positiva $M_{1}$, ou seja,

$$
\|u\| \leq M_{1} .
$$

Observe que em [6], a prova depende de uma solução explicita, especificamente, no caso de tração uniforme, $u\left(x_{1}, x_{2}\right)=\tau\left(x_{2}\right)^{2} h / 2$ e no caso do cisalhamento uniforme, $u\left(x_{1}, x_{2}\right)=\tau x_{1} x_{2}$. Nestes exemplos, as soluções são limitadas em $\Omega$.

\section{Problema Modificado}

Consideremos agora uma outra situação, em que a região $\Omega$ é submetida à mesma distribuição de tensões no trecho da fronteira $\partial \Omega \backslash L_{0}$, onde $L_{0}=\left\{\left(x_{1}, x_{2}\right) \mid x_{1}=\right.$ $\left.0,-h / 2<x_{2}<h / 2\right\}$; mas em $L_{0}$, temos agora, uma distribuição de tensões modificadas $f$ e $g$ em lugar de $f_{1}$ e $g_{1}$ que mantém o corpo em equilíbrio. Portanto, as condições (2.17) a (2.19) são inalteradas e, em lugar de (2.20) na formulação do Problema referencial, temos:

$$
\phi\left(0, x_{2}\right)=\int_{-h / 2}^{x_{2}}\left(\int_{-h / 2}^{t} f(s) d s\right) d t, \quad \phi,,_{1}\left(0, x_{2}\right)=-\int_{-h / 2}^{x_{2}} g(s) d s .
$$


Denotaremos por $\phi\left(x_{1}, x_{2}\right)$ as soluções do problema $(2.16),(2.17)-(2.19)$ e $(3.2)$ substituindo as condições (2.20), que passaremos a denominar Problema modificado.

Neste trabalho estamos interessados nas distribuições de tensões $\phi\left(x_{1}, x_{2}\right)$ que estão a uma distância finita de $u\left(x_{1}, x_{2}\right)$, isto é, que satisfaçam

$$
\|\phi-u\| \leq M_{2}, \text { para alguma constante } M_{2} \geq 0
$$

desde que $\lambda_{1}, \lambda_{2}$ e $\lambda_{3}$ sejam suficientementes pequenos.

Estamos aí supondo um comportamento de dependência contínua em relação aos parâmetros para a solução $\phi$.

\section{Energia do Problema}

O objetivo central deste trabalho é demonstrar que a solução $\phi\left(x_{1}, x_{2}\right)$ do Problema modificado, que nos dá a distribuição das tensões em $\Omega$ quando modificamos os dados na fronteira em $L_{0}$ do Problema referencial, decai exponencialmente para a solução $u\left(x_{1}, x_{2}\right)$, para $0 \ll x_{1}$. Isto é o que se pretende afirmar quando dizemos que "longe de $L_{0}$ as duas soluções estão próximas". Em outras palavras, o Princípio de Saint-Venant é verdadeiro no contexto da elasticidade não linear aqui abordado.

Com esse objetivo, mostraremos que a energia definida por

$$
E(z)=\int_{\Omega_{z}} \rho(\phi) w,{ }_{i j} w,{ }_{i j} d A
$$

com

$$
w\left(x_{1}, x_{2}\right)=\phi\left(x_{1}, x_{2}\right)-u\left(x_{1}, x_{2}\right)
$$

$\mathrm{e}$

$$
\Omega_{z}=\left\{\left(x_{1}, x_{2}\right) \mid 0<z<x_{1}<l,-\frac{h}{2}<x_{2}<\frac{h}{2}\right\},
$$

decai exponencialmente para zero quando $z \rightarrow l$.

Observe que $\rho(\phi) \geq 1-\left|\lambda_{1}\right|\|\phi\|-\left|\lambda_{2}\right|\|\phi\|^{2}-\left|\lambda_{3}\right|\|\phi\|^{2}$, por (3.1), (3.3) e desigualdade triangular obtemos $\|\phi\| \leq\|\phi-u\|+\|u\| \leq M_{1}+M_{2}$, segue portanto que $\rho(\phi) \geq 1-\left|\lambda_{1}\right|\left(M_{1}+M_{2}\right)-\left(\left|\lambda_{2}\right|+\left|\lambda_{3}\right|\right)\left(M_{1}+M_{2}\right)^{2}$. Assim, para $\left|\lambda_{1}\right|,\left|\lambda_{2}\right|$ e $\left|\lambda_{3}\right|$ suficientemente pequenos, podemos tomar $\rho(\phi) \geq \delta>0$. Por exemplo, para

$$
\left|\lambda_{1}\right| \leq 1 / 6\left(M_{1}+M_{2}\right), \quad\left|\lambda_{2}\right| \leq 1 / 6\left(M_{1}+M_{2}\right)^{2} \quad \text { e } \quad\left|\lambda_{3}\right| \leq 6\left(M_{1}+M_{2}\right)^{2}
$$

obtemos

$$
\rho(\phi) \geq 1 / 2
$$

\section{Resultado Principal}

Teorema 4.1. Seja $u\left(x_{1}, x_{2}\right)$ a solução do Problema referencial $(2.16),(2.17)-$ $(2.20)$, e $\phi\left(x_{1}, x_{2}\right)$ a solução do Problema modificado (2.16), (2.17) - (2.19), (3.2). Suponhamos que $\rho(\phi)=1+\mathcal{H}(\phi)$, com

$$
\mathcal{H}(\phi)=\lambda_{1} \phi,{ }_{k k}+\lambda_{2} \phi,{ }_{k k} \phi, l l+\lambda_{3} \phi, k l \phi, k l,
$$


e que $\|u\| \leq M_{1}$ e $\|\phi-u\| \leq M_{2}$. Então, existem $\delta_{1}>0$ e $\delta_{2}>0$ tal que, se $\left|\lambda_{1}\right| \leq \delta_{1},\left|\lambda_{2}\right| \leq \delta_{2} e\left|\lambda_{3}\right| \leq \delta_{2}$, a energia definida em (3.4) satisfaz à desigualdade

$$
E(z) \leq(1+D) E(0) e^{-z / D}, \quad 0 \leq z \leq l,
$$

onde $E(0)$ é uma constante positiva, e $D>1$ é uma constante que depende da geometria do problema, e de $\lambda_{1}, \lambda_{2}$ e $\lambda_{3}$.

Demonstração: Usando as condições de fronteira e a integração por partes, podemos reescrever a energia $E(z)$ da seguinte maneira,

$$
E(z)=-\int_{L_{z}} \rho w,{ }_{11} w,{ }_{1} d x_{2}-2 \int_{L_{z}} \rho w,{ }_{12} w,{ }_{2} d x_{2}+\int_{L_{z}}\left(\rho w,{ }_{11}\right),{ }_{1} w d x_{2}+N
$$

onde

$$
N=-\int_{\Omega_{z}}\left[\left(1+\lambda_{1} \phi,{ }_{k k}+\lambda_{2} \phi,{ }_{k k} \phi, l l+\lambda_{3} \phi, k l \phi, k l\right) u,{ }_{i j}\right],{ }_{i j} w d A .
$$

Por (3.5), temos por exemplo que $\phi, k k=w, k k+u, k k$, substituindo na identidade (4.3), passamos a ter na energia $E(z)$, as integrais envolvendo apenas $w$ e $u$. Observe que as integrais são em $L_{z}$ ou $\Omega_{z}$.

Desenvolvendo os termos das integrais acima e usando a definição da energia e da identidade

$$
E^{\prime}(z)=-\int_{L_{z}} \rho(\phi) w,{ }_{i j} w,{ }_{i j} d A
$$

estimamos as integrais ora pela energia ora por sua derivada da seguinte maneira:

i) Integrais em $\Omega_{z}$

$$
\int_{\Omega_{z}}\left[(w, k k) u,{ }_{11}\right] w,{ }_{11} d A \leq K \int_{\Omega_{z}}\left|w,{ }_{11}\right|\left|w,,_{11}\right| d A+K \int_{\Omega_{z}}\left|w,,_{22}\right|\left|w,,_{11}\right| d A
$$

onde $K=M_{1}+M_{2}$, e $M_{1}, M_{2}$ são as constantes em (3.1) e (3.3). Aplicando a desigualdade de Schwarz a cada uma das duas integrais anteriores, utilizando que $\rho(\phi) \geq 1 / 2$ obtido em (3.7), e a seguir a definição da energia, obtemos

$$
\int_{\Omega_{z}}\left|w,{ }_{11}\right|\left|w,{ }_{11}\right| d A \leq\left[2 \int_{\Omega_{z}} \rho(w, 2) d A\right]^{1 / 2}\left[2 \int_{\Omega_{z}} \rho\left(w,{ }_{11}^{2}\right) d A\right]^{1 / 2} \leq 2 E(z),
$$

e analogamente,

$$
\int_{\Omega_{z}}\left|w,{ }_{22}\right|\left|w,{ }_{11}\right| d A \leq 2 E(z) .
$$

Temos, portanto, que

$$
\int_{\Omega_{z}}\left[(w, k k) u,{ }_{11}\right] w,{ }_{11} d A \leq 4 K E(z) .
$$

ii) Integrais em $L_{z}$ 
Usando a desigualdade de Poincaré-Friedrichs (ver [8], [12]), a desigualdade de Schwarz e o fato que $\rho \geq 1 / 2$, obtemos

$$
\int_{L_{z}}\left|w,{ }_{11}\right||w| d x_{2} \leq\left[2 \int_{L_{z}} \rho\left(w, 2{ }_{11}\right) d x_{2}\right]^{1 / 2}\left[2 A \int_{L_{z}} \rho\left(w_{22}^{2}\right) d x_{2}\right]^{1 / 2}
$$

Logo,

$$
\int_{L_{z}}\left|w,_{11}\right||w| d x_{2} \leq 2 A^{\frac{1}{2}}\left(-E^{\prime}(z)\right)
$$

Usando estas estimativas e outras semelhantes, obtemos a desigualdade

$$
\begin{aligned}
\alpha \int_{z}^{l} E(s) d s \leq & A^{1 / 2}\left[4(2 K)^{1 / 2}+4 K\left(3\left|\lambda_{1}\right|+9 K\left(\left|\lambda_{2}\right|+18 K\left|\lambda_{3}\right|\right)\right)\right] E(z) \\
& -A^{1 / 2}\left[(2 K)^{1 / 2}+4 K\left(\left|\lambda_{1}\right|+6 K\left(\left|\lambda_{2}\right|+15 K\left|\lambda_{3}\right|\right)\right)\right] E^{\prime}(z)
\end{aligned}
$$

onde $\alpha=1-12 K\left(\left|\lambda_{1}\right|+6 K\left(\left|\lambda_{2}\right|+\left|\lambda_{3}\right|\right)\right)$.

Definindo $B=A^{1 / 2}\left[4(2 K)^{1 / 2}+4 K\left(3\left|\lambda_{1}\right|+9 K\left(\left|\lambda_{2}\right|+18 K\left|\lambda_{3}\right|\right)\right)\right]$, segue, da desigualdade (4.4), que

$$
\alpha \int_{z}^{l} E(s) d s \leq B E(z)-B E^{\prime}(z) .
$$

Tomando $\left|\lambda_{1}\right|<1 /\left(6^{2} K\right),\left|\lambda_{2}\right|<1 /\left(6^{3} K^{2}\right)$ e $\left|\lambda_{3}\right|<1 /\left(6^{3} K^{2}\right)$, as desigualdades em (3.6) são satisfeitas. Definindo agora $\delta_{1}=1 /\left(6^{2} K\right)$ e $\delta_{2}=1 /\left(6^{3} K^{2}\right)$, segue que para todo $\left|\lambda_{1}\right|<\delta_{1}$ e $\left|\lambda_{2}\right|,\left|\lambda_{3}\right|<\delta_{2}$, obtemos que $12 K\left(\left|\lambda_{1}\right|+6 K\left(\left|\lambda_{2}\right|+\left|\lambda_{3}\right|\right)\right)<1$. Obtemos assim $0<\alpha=1-12 K\left(\left|\lambda_{1}\right|+6 K\left(\left|\lambda_{2}\right|+\left|\lambda_{3}\right|\right)\right)$. Se necessário, é possível obter intervalos para $\lambda_{1}, \lambda_{2}$ e $\lambda_{3}$ tais que $\alpha \geq \eta>0$, onde $\eta$ é dado.

Dividindo (4.5) por $\alpha$, adicionando $E(z)$ a ambos os lados da desigualdade e $-E^{\prime}(z)>0$ somente no lado direito, podemos, então, escrever

$$
E(z)+\int_{z}^{l} E(s) d s \leq(B / \alpha+1)\left(E(z)-E^{\prime}(z)\right) .
$$

Definindo, agora,

$$
H(z)=E(z)+\int_{z}^{l} E(s) d s
$$

obtemos $H^{\prime}(z)=E^{\prime}(z)-E(z)$, e daí,

$$
1 / D \leq-H^{\prime}(z) / H(z), \quad \text { onde } D=\frac{B}{\alpha}+1>0 .
$$

Integrando (4.7) de 0 a $z$, obtemos

$$
H(z) \leq H(0) e^{-z / D}
$$

O que resta é estimar $H(0)$ em termos de constantes conhecidas. Para isso, substituindo em (4.8) o valor de $H(z)$, temos

$$
E(z)+\int_{z}^{l} E(s) d s \leq H(0) e^{-z / D} .
$$


Daí, por integração, obtemos

$$
H(0)=E(0)+\int_{0}^{l} E(s) d s \leq\left(1+\frac{1-e^{-k l}}{1 / D+e^{-k l}}\right) E(0)<(1+D) E(0) .
$$

Substituindo esta estimativa em (4.8), e observando que $E(z) \leq H(z)$, obtemos

$$
E(z) \leq(1+D) E(0) e^{-z / D},
$$

que é o que queríamos demonstrar.

\title{
5. Consideração Final
}

O resultado central deste trabalho, o Teorema 4.1, fazendo uso da hipótese (3.1), $\|u\| \leq M_{1}$, juntamente com a hipótese (3.3), $\|\phi-u\| \leq M_{2}$, onde $M_{1}$ e $M_{2}$ são constantes positivas, consegue unificar não só a tração uniforme cuja solução do problema referencial é $u\left(x_{1}, x_{2}\right)=\tau\left(x_{2}\right)^{2} h / 2$, e o cisalhamento uniforme $u\left(x_{1}, x_{2}\right)=$ $\tau x_{1} x_{2}$, apresentadas por Horgan e Payne [6], mas também outras situações de distribuição de forças na fronteira de $\Omega$, tais como as soluções do problema de flexão dada por $u\left(x_{1}, x_{2}\right)=\tau\left(x_{2}^{3}-x_{1}^{3}\right) / 6$ ou $u\left(x_{1}, x_{2}\right)=\tau\left(x_{2}^{3}-3 x_{1}^{2} x_{2}\right) / 6$.

\begin{abstract}
.
This work extends the results obtained by Horgan e Payne [6] in 1992. We show that Saint-Venant Principle is valid for a plane retangular region occupied by an elastic body, whose constitutive equation contains non-linear terms as a generalization of the Hooke's law for small deformations. The non-linear terms considered by Horgan e Payne [6] are at least of the third order. The inclusion of second order terms in this paper provides a more realistic model for the theory. The main result is established using techniques of differential inequalities for quadratic functionals.
\end{abstract}

\section{Referências}

[1] J. Boussinesq, "Application des potentiels à l'étude de l'équilibre et des mouvements des solides élastiques", Gauthier-Villars, Paris, 1885.

[2] S. Breuer e J.J. Roseman, On Saint-Venant's principle in three di-mensional nonlinear elasticity, Arch. Rational Mech. Anal., 80 (1977), 191-203.

[3] C.O. Horgan e J.K. Knowles, The effect of nonlinearity on a principle of SaintVenant type, J. Elasticity, 11 (1981), 271-291.

[4] C.O. Horgan e L.E. Knowles, Recent developments concerning Saint-Venant's principle, Adv. Appl. Mech., 23 (1983), 179-269.

[5] C.O. Horgan e L.E. Payne, On Saint-Venant's principle in finite anti-plane shear: an energy approach, Arch. Rational Mech. Anal., 109 (1990), 107-137. 
[6] C.O. Horgan e L.E. Payne, A Saint-Venant principle for a theory of nonlinear plane elasticity, Quart. Appl. Math., 4 (1992), 641-675.

[7] J.D. Silva, "O Princípio de Saint-Venant em Elasticidade Não Linear", Tese de Doutorado IM, UFRJ, Rio de Janeiro, RJ, 2002.

[8] L.A. Medeiros e M.M. Miranda, "Espaços de Sobolev e Equações Diferenciais Parciais", IM, UFRJ, Rio de Janeiro, 1993.

[9] J.J. Roseman, The principle of Saint-Venant in linear and nonlinear plane elasticity, Arch. Rational Mech. Anal., 26 (1967), 142-162.

[10] A.B. Saint-Venant, Mémoire sur la torsion des prismes, l'Academie des Sciences de l'Institut Impérial de France, 14 (1853), 233-560.

[11] R.A Toupin, Saint-Venant's Principle, Arch. Rational Mech. Anal., 18 (1965), 83-96.

[12] P. Villaggio, "Qualitative Methods in Elasticity", Noordhoff Intern. Publishing, Leyden-Pisa, 1977. 
